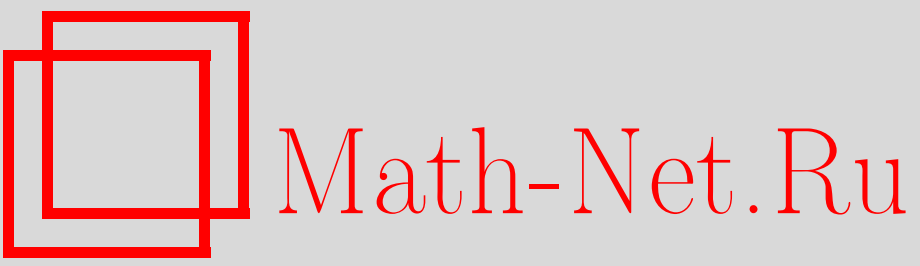

В. А. Москаленко, Электрон-фононное взаимодействие сильно скоррелированных систем, ТМФ, 1997, том 111, номер 3, 439-451

DOI: https://doi.org/10.4213/tmf1020

Использование Общероссийского математического портала Math-Net.Ru подразумевает, что вы прочитали и согласны с пользовательским соглашением

http://www.mathnet.ru/rus/agreement

Параметры загрузки:

IP: 54.197 .130 .99

26 апреля 2023 г., 14:54:04 
ТЕОРЕТИЧЕСКАЯ

И МАТЕМАТИЧЕСКАЯ

ФИЗИКА

Том 111, № 3

июнь, 1997

В. А. Москаленко*

\title{
ЭЛЕКТРОН-ФОНОННОЕ ВЗАИМОДЕЙСТВИЕ СИЛЬНО СКОРРЕЛИРОВАННЫХ СИСТЕМ
}

\begin{abstract}
Модель Хаббарда, дополненная взаимодействием Холстейна локализованных электронов и оптических бездисперсных фононов, при помощи канонического преобразования сводится к модели, в которой происходят перескоки поляронов, сопровождаемые перескоками неограниченного числа фононов. Это обстоятельство и тот факт, что имеется сильное одноузельное взаимодействие электронов, присущее модели Хаббарда, приводят к необходимости формулировки новой диаграммной техники. Такая техника характеризуется неприводимыми одноузельными многочастичными функциями Грина или кумулянтами Кубо. Присутствие фононов вызывает существенную перенормировку как одночастичной, так и многочастичных функций Грина. Для ренормированной электронной функции Грина сформулировано уравнение Дайсона, тогда как для фононной функции этого сделать не удается из-за множественности фононов, которые участвуют в процессах перескока. Показана справедливость теоремы о связанных диаграммах.
\end{abstract}

\section{ВВЕДЕНИЕ}

Вопрос о роли электрон-фононного взаимодействия для систем с сильными электронными корреляциями является одним из центральных. Он актуален особенно для купратов, в которых наблюдается высокотемпературная сверхпроводимость. Сами по себе фононы, по-видимому, не могут вызвать сверхпроводимость в этих материалах, однако они могут оказать влияние на установление сверхпроводящего перехода и изотопическую зависимость температуры этого перехода. В настояшей работе исследуется нормальное состояние системы с сильными корреляциями при наличии взаимодействия электронов с колебаниями кристаллической решетки. Делается попытка учесть влияние фононов на электронные свойства и влияние сильного электронного взаимодействия на свойства фононов. Используются формализм мацубаровских функций Грина и новый диаграммный подход, разработанньй в работах [1-3].

\section{1. ОСНОВНЫЕ ОПРЕДЕЛЕНИЯ}

Рассмотрим модель Хаббарда [4] сильно скоррелированных электронов, дополненную локальным взаимодействием Холстейна [5] оптических фононов, принадлежащих

* Институт прикладной физики АН Молдовы, Кишинев, Молдова 
к определенному узлу кристаллической решетки с локальной плотностью электрического заряда. Гамильтониан системы имеет вид

$$
H=H_{\mathrm{e}}+H_{\mathrm{e}-\mathrm{ph}}+H_{\mathrm{ph}}^{0}
$$

где

$$
\begin{aligned}
H_{\mathrm{e}} & =\sum_{i j \sigma} t(j-i) c_{j \sigma}^{+} c_{i \sigma}-\mu \sum_{i \sigma} c_{i \sigma}^{+} c_{i \sigma}+U \sum_{i} n_{i \uparrow} n_{i \downarrow}, \\
H_{\mathrm{e}-\mathrm{ph}} & =g \sum_{i} q_{i} n_{i}, \quad H_{\mathrm{ph}}^{0}=\hbar \omega_{0} \sum_{i} b_{i}^{+} b_{i}, \quad n_{i}=\sum_{\sigma} n_{i \sigma},
\end{aligned}
$$

здесь $c_{i \sigma}^{+}\left(c_{i \sigma}\right)$ - оператор рождения (уничтожения) электрона со спином $\sigma$ на узле $i$ кристаллической решетки, $n_{i \sigma}=c_{i \sigma}^{+} c_{i \sigma}-$ плотность числа электронов с данным спином, $t(j-i)$ - интеграл перескока электронов, $\mu$ - химический потенциал системы, $U-$ кулоновское взаимодействие электронов на узле, $b_{i}^{+}\left(b_{i}\right)$ - операторы рождения (уничтожения) локальных фононов, обладающих бездисперсной частотой колебаний $\omega_{0}, g-$ константа электрон-фононного взаимодействия, $q_{i}, p_{i}$ - локальная координата и импульс фонона:

$$
q_{i}=\frac{1}{\sqrt{2}}\left(b_{i}+b_{i}^{+}\right), \quad p_{i}=\frac{i}{\sqrt{2}}\left(b_{i}^{+}-b_{i}\right) .
$$

Температурные функции Грина электронов и фононов равны

$$
\begin{aligned}
G\left(\vec{x}, \sigma, \tau \mid \vec{x}^{\prime} \sigma^{\prime} \tau^{\prime}\right) & =-\left\langle T \hat{c}_{\vec{x} \sigma}(\tau) \hat{\bar{c}}_{\vec{x}^{\prime} \sigma^{\prime}}\left(\tau^{\prime}\right)\right\rangle \\
D\left(\vec{x}, \tau \mid \vec{x}^{\prime}, \tau^{\prime}\right) & =-\left\langle T \hat{q}_{\vec{x}}(\tau) \tilde{q}_{\vec{x}^{\prime}}\left(\tau^{\prime}\right)\right\rangle
\end{aligned}
$$

где операторы со шляпкой написаны в гейзенберговском представлении. Для исключения линейного по координатам фононов взаимодействия выполним над гамильтонианом (1) унитарное преобразование Ланга-Фирсова [6]:

$$
\widetilde{H}=e^{S} H e^{-S}, \quad S=-i \bar{g} \sum_{i} n_{i} p_{i}, \quad \bar{g}=\frac{g}{\hbar \omega_{0}} .
$$

Преобразованные операторы отмечаются знаком тильда:

$$
\begin{gathered}
\tilde{b}_{i}=b_{i}-\frac{1}{\sqrt{2}} \bar{g} n_{i}, \quad \tilde{b}_{i}^{+}=b_{i}-\frac{1}{\sqrt{2}} \bar{g} n_{i}, \quad \tilde{q}_{i}=q_{i}-\bar{g} n_{i}, \\
\tilde{c}_{i \sigma}=c_{i \sigma} e^{i \bar{g} p_{i}}, \quad \tilde{c}_{i \sigma}^{+}=c_{i \sigma}^{+} e^{-i \bar{g} p_{i}} .
\end{gathered}
$$

В результате унитарного преобразования возникает гамильтониан

$$
\widetilde{H}=H_{\mathrm{e}}^{0}+H_{\mathrm{ph}}^{0}+H_{\mathrm{int}}, \quad H_{\mathrm{e}}^{0}=\sum_{i} H_{i \mathrm{e}}^{0},
$$

где

$$
\begin{array}{ll}
H_{i \mathrm{e}}^{0}=-\mu \sum_{\sigma} n_{\vec{i} \sigma}+\widetilde{U} n_{\vec{i} \uparrow} n_{\vec{i} \downarrow}, & \tilde{\mu}=\mu+g^{2} / 2 \hbar \omega_{0}, \\
H_{\mathrm{int}}=\sum_{i j \sigma} t(\vec{j}-\vec{i}) \tilde{c}_{\vec{j} \sigma}^{+} \tilde{c}_{\vec{i} \sigma}, & \tilde{U}=U-g^{2} / \hbar \omega_{0} .
\end{array}
$$


В нулевом приближении, когда пренебрегается перескоками электронов, имеются системы фононов и сильно взаимодействующих электронов на узле решетки. Оператор $H_{\text {int }}$ соответствует туннелированию электронов, окруженных облаком фононов, т.е. поляронов. Локальное электрон-фононное взаимодействие (2) привело к возникновению в $\widetilde{H}$ притягиваюшего взаимодействия электронов, что уменьшило их исходное отталкивание, и к перенормировке химического потенциала. Выполним в (4) унитарное преобразование операторов путем введения единичного оператора $e^{S} e^{-S}$ под знаком следа и циклически переставим $e^{S}$. В результате в (4) возникают операторы $\tilde{c}^{+}, \tilde{c}$ и $\tilde{q}$. В представлении взаимодействия определения (4) приобретают вид

$$
\begin{aligned}
G\left(\vec{x} \sigma \tau \mid \vec{x}^{\prime} \sigma^{\prime} \tau^{\prime}\right) & =-\left\langle T \tilde{c}_{\vec{x} \sigma}(\tau) \tilde{\bar{c}}_{\vec{x}^{\prime} \sigma^{\prime}}\left(\tau^{\prime}\right) U(\beta)\right\rangle_{0}\langle U(\beta)\rangle_{0}^{-1} \\
D\left(\vec{x} \tau \mid \vec{x}^{\prime}, \tau^{\prime}\right) & =-\left\langle T \tilde{q}_{\vec{x}}(\tau) \tilde{q}_{\vec{x}^{\prime}}\left(\tau^{\prime}\right) U(\beta)\right\rangle_{0}\langle U(\beta)\rangle_{0}^{-1}
\end{aligned}
$$

где оператор эволюции $U(\beta)$ равен

$$
\begin{aligned}
U(\beta) & =T \exp \left(-\int_{0}^{\beta} H_{\mathrm{int}}(\tau) d \tau\right)= \\
& =\sum_{n=0}^{\infty} \frac{(-1)^{n}}{n !} \int_{0}^{\beta} d \tau_{1} \cdots \int_{0}^{\beta} d \tau_{n} T H_{\mathrm{int}}\left(\tau_{1}\right) \ldots H_{\mathrm{int}}\left(\tau_{n}\right) .
\end{aligned}
$$

Так как оператор взаимодействия $H_{\text {int }}$ выражается через поляронные операторы, то формула (9) для электронной функции Грина является более удобной, чем исходное выражение (4). Что касается фононной функции, то ее можно переписать в виде

$$
D\left(x \mid x^{\prime}\right)=B_{1}\left(x \mid x^{\prime}\right)+\bar{g} C\left(x \mid x^{\prime}\right)+\bar{g} C\left(x^{\prime} \mid x\right)+\bar{g}^{2} A\left(x \mid x^{\prime}\right),
$$

где

$$
\begin{aligned}
B_{1}\left(x \mid x^{\prime}\right) & =-\left\langle T q_{\vec{x}}(\tau) q_{\vec{x}^{\prime}}\left(\tau^{\prime}\right) U(\beta)\right\rangle_{0}\langle U(\beta)\rangle_{0}^{-1}, \\
C\left(x \mid x^{\prime}\right) & =-\left\langle T q_{\vec{x}}(\tau) n_{\vec{x}^{\prime}}\left(\tau^{\prime}\right) U(\beta)\right\rangle_{0}\langle U(\beta)\rangle_{0}^{-1} \\
A\left(x \mid x^{\prime}\right) & =-\left\langle T n_{\vec{x}}(\tau) n_{\vec{x}^{\prime}}\left(\tau^{\prime}\right) U(\beta)\right\rangle_{0}\langle U(\beta)\rangle_{0}^{-1} .
\end{aligned}
$$

Здесь и в дальнейшем используется сокрашенное обозначение индексов операторов: $x=(\vec{x}, \sigma, \tau)$ для электронов и $x=(\vec{x}, \tau)$ для фононов.

Наряду с этими функциями существует величина

$$
Q(x)=\left\langle T q_{\vec{x}}(\tau) U(\beta)\right\rangle_{0}\langle U(\beta)\rangle_{0}^{-1},
$$


равная равновесному положению фононов. Возникающие в процессе ее вычисления фононные средние имеют вид

$$
\begin{aligned}
&\left\langle T \exp \left\{i \bar{g}\left(p\left(\tau_{1}\right)-p\left(\tau_{2}\right)\right)\right\}\right\rangle_{0}= \exp \left[-\frac{\bar{g}^{2}}{2}\left\langle T\left(p\left(\tau_{1}\right)-p\left(\tau_{2}\right)\right)^{2}\right\rangle_{0}\right], \\
&\left\langle T q(\tau) \exp \left\{i \bar{g}\left(p\left(\tau_{1}\right)-p\left(\tau_{2}\right)\right)\right\}\right\rangle_{0}= i \bar{g}\left\langle T q(\tau)\left(p\left(\tau_{1}\right)-p\left(\tau_{2}\right)\right)\right\rangle_{0} \times \\
& \times\left\langle T \exp \left\{i \bar{g}\left(p\left(\tau_{1}\right)-p\left(\tau_{2}\right)\right)\right\}\right\rangle_{0}, \\
&\left\langle T q(\tau) q\left(\tau^{\prime}\right) \exp \left\{i \bar{g}\left(p\left(\tau_{1}\right)-p\left(\tau_{2}\right)\right)\right\}\right\rangle_{0}= {\left[\left\langle T q(\tau) q\left(\tau^{\prime}\right)\right\rangle_{0}-\bar{g}^{2}\left\langle T q(\tau)\left(p\left(\tau_{1}\right)-p\left(\tau_{2}\right)\right)\right\rangle_{0} \times\right.} \\
&\left.\times\left\langle T q\left(\tau^{\prime}\right)\left(p\left(\tau_{1}\right)-p\left(\tau_{2}\right)\right)\right\rangle_{0}\right]\left\langle T \exp \left\{i \bar{g}\left(p\left(\tau_{1}\right)-p\left(\tau_{2}\right)\right)\right\}\right\rangle_{0}, \\
&\left\langle T\left(p(\tau)-p\left(\tau^{\prime}\right)\right)^{2}\right\rangle_{0}= \bar{N}\left(1-\exp \left\{-\hbar \omega_{0}\left|\tau-\tau^{\prime}\right|\right\}\right) \times \\
& \times\left[\exp \left\{\beta \hbar \omega_{0}\right\}-\exp \left\{\hbar \omega_{0}\left|\tau-\tau^{\prime}\right|\right\}\right], \\
&\left\langle T q(\tau) q\left(\tau^{\prime}\right)\right\rangle_{0}= \frac{\bar{N}}{2}\left[\exp \left\{\hbar \omega_{0}\left|\tau-\tau^{\prime}\right|\right\}+\exp \left\{\hbar \omega_{0}\left(\beta-\left|\tau-\tau^{\prime}\right|\right)\right\}\right], \\
& \bar{N}=\left(\exp \left\{\beta \hbar \omega_{0}\right\}-1\right)^{-1} .
\end{aligned}
$$

Усреднение в формулах (9) выполняется по статистическому оператору

$$
e^{-\beta H^{0}}=\prod_{i} e^{-\beta H_{i \mathrm{e}}^{0}} e^{-\beta H_{i \mathrm{ph}}^{0}}
$$

факторизованному по узлам решетки. Фононный оператор диагонален в свободных фононных операторах, тогда как электронный узельный гамильтониан, содержаший $\widetilde{U}$, диагонализуется в операторах Хаббарда [4]. Относительно величины $\widetilde{U}$ и ее знака не делается специальных предположений, кроме того, что она существенна и должна быть учтена уже в нулевом приближении теории. Термодинамическая теория возмушений по величине $H_{\text {int }}$ нуждается в обобщении диаграммной техники в силу того, что опе-

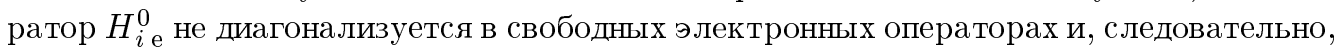
теорема Вика для статистических средних от произведения электронных операторов не работает. Для фононных операторов справедлива обычная теорема Вика, однако в силу того, что туннелирование между узлами решетки осушествляется не электронами, а поляронами, эта часть статистических усреднений также нуждается в модификациях, суть которых определена формулами (14).

\section{2. ТЕОРИЯ ВОЗМУЩЕНИЙ}

Теория возмушений по оператору взаимодействия $H_{\text {int }}$ приводит к необходимости вычисления статистических средних от произведения операторов

$$
t(\overline{1}-1) \ldots t(\bar{n}-n)\left\langle T \tilde{\bar{c}}_{T} \tilde{c}_{1} \ldots \tilde{\bar{c}}_{\bar{n}} \tilde{c}_{n}\right\rangle_{0}
$$

где $\tilde{c}_{i}, \tilde{\bar{c}}_{i}$ определены формулами (6). Здесь для краткости один индекс $n$ обозначает совокупность узельного, спинового и временного индексов. Чтобы выполнить усреднение (16) в локальном нулевом приближении, следует под символом $T$-произведения 
объединить с учетом знака перестановки все электронные операторы, имеющие одинаковый узельный индекс. Тем самым мы разбиваем произведение (16) на группы операторов так, чтобы внутри каждой группы узлы были одинаковы, но одна группа отличалась бы от другой узельным индексом. Далее необходимо усреднить вместе те из операторов, которые обладают одинаковым узельным индексом, передав им из статистического оператора (15) множитель соответствуюшего узла. Для выполнения этого разбиения введем необходимое число узельных $\delta_{i j}$-символов Кронекера, соответствуюших равенству узлов, и символов $\psi_{i j}=1-\delta_{i j^{\prime}}$, отвечающих их неравенству. Это можно сделать, домножив произведение (16) любым числом единиц:

$$
1=\prod_{i j}\left(\delta_{i j}+\psi_{i j}\right)
$$

Так как электронные операторы не заменяются на этом этапе вычисления суммами операторов Хаббарда, то проблема знака новых возникающих групп операторов легко решается. При образовании всевозможных групп операторов с одинаковыми индексами узлов следует соблюдать два правила, а именно: 1) числа электронных операторов рождения и уничтожения (пока речь не идет о сверхпроводимости) должны быть одинаковыми и 2 ) в одну и ту же группу не входят индексы $\vec{i}$ и $\vec{j}$, принадлежащие одному интегралу перескока в силу его свойства $t(0)=0$. Это последнее свойство может быть выражено присутствием символов $\psi_{\bar{i}}$. Приведем простой пример. Рассмотрим операторное выражение, возникающее во втором порядке теории возмушений по $H_{\text {int }}$ :

$$
\psi_{\overline{1} 1} \psi_{\overline{2} 2}\left\langle T \tilde{\bar{c}}_{\overline{1}} \tilde{c}_{1} \tilde{\bar{c}}_{\overline{2}} \tilde{c}_{2}\right\rangle_{0}
$$

Введем в этом выражении рядом с $\psi_{\overline{1} 1} \psi_{\overline{2} 2}$ произведение двух единищ:

$$
\left(\delta_{1 \overline{2}}+\psi_{1 \overline{2}}\right)\left(\delta_{2 \overline{1}}+\psi_{2 \overline{1}}\right)=\delta_{1 \overline{2}} \delta_{2 \overline{1}}+\psi_{1 \overline{2}} \delta_{2 \overline{1}}+\psi_{2 \overline{1}} \delta_{1 \overline{2}}+\psi_{1 \overline{2}} \psi_{2 \overline{1}}
$$

Из всех слагаемых в последнем выражении следует сохранить только первое, т.к. остальные три дадут нули при последуюшем статистическом усреднении $\tilde{c}$-операторов. Следовательно, можно написать

$$
\psi_{\overline{1} 1} \psi_{\overline{2} 2}\left\langle T \tilde{\bar{c}}_{\overline{1}} \tilde{c}_{1} \tilde{\bar{c}}_{\overline{2}} \tilde{c}_{2}\right\rangle_{0}=-\psi_{\overline{1} 1} \psi_{\overline{2} 2}\left\langle T\left(\tilde{c}_{1} \tilde{\bar{c}}_{\overline{2}}\right)\left(\tilde{c}_{2} \tilde{\bar{c}}_{\overline{1}}\right)\right\rangle_{0} \delta_{1 \overline{2}} \delta_{2 \overline{1}}
$$

Здесь круглая скобка объединяет операторы одного узла и эти узлы для обеих групп разные. После этих разъяснений получаем статистическое среднее вида

$$
\left.\psi_{\overline{1} 1} \psi_{\overline{2} 2}\left\langle T \tilde{\bar{c}}_{\overline{1}} \tilde{c}_{1} \tilde{\bar{c}}_{\overline{2}} \tilde{c}_{2}\right\rangle_{0}=-\psi_{\overline{1} 1} \psi_{\overline{2} 2}\left\langle T \tilde{c}_{1} \tilde{\bar{c}}_{\overline{2}}\right\rangle_{0}\left\langle T \tilde{c}_{2} \tilde{\bar{c}}_{\overline{1}}\right)\right\rangle_{0}
$$

В этом выражении можно опустить $\delta$-символы Кронекера $\delta_{1 \overline{2}}, \delta_{2 \overline{1}}$, т.к. они содержатся в определении нулевых функций Грина:

$$
\begin{aligned}
& \widetilde{G}^{0}(1 \mid \overline{2})=-\left\langle T \tilde{c}_{1} \tilde{\bar{c}}_{\overline{2}}\right\rangle_{0}=G^{0}(1 \mid \overline{2})\left\langle\exp \left\{i \bar{g}\left(p\left(\tau_{1}\right)-p\left(\tau_{2}\right)\right)\right\}\right\rangle_{0} \\
& G^{0}(1 \mid \overline{2})=-\left\langle T c_{1} \bar{c}_{\overline{2}}\right\rangle_{0}=-\delta_{i_{1} j_{2}}\left\langle T c_{\sigma_{1}}\left(\tau_{1}\right) \bar{c}_{\sigma_{2}}\left(\tau_{2}\right)\right\rangle_{0}
\end{aligned}
$$




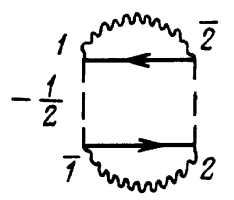

Рис. 1. Простейшая вакуумная диаграмма.

Одноузельные нулевые функции Грина (19) изображаются графически прямыми направленными линиями, соединяюшими две точки 1 и $\overline{2}$, у которых узельные индексы совпадают, однако временные аргументы различны.

Вакуумная диаграмма второго порядка теории возмущений имеет вид, приведенный на рис. 1. На этом рисунке интеграл перескока $t(i-j)$ изображается штриховой линией, соединяюшей два узла $i$ и $j$ решетки, волнистыми линиями изображены статистические средние от следующих фононных множителей:

$$
\left\langle T \exp \left\{i \bar{g}\left(p_{1}\left(\tau_{1}\right)-p_{1}\left(\tau_{2}\right)\right)\right\}\right\rangle_{0}\left\langle T \exp \left\{i \bar{g}\left(p_{2}\left(\tau_{2}\right)-p_{2}\left(\tau_{1}\right)\right)\right\}\right\rangle_{0}
$$

В третьем порядке теории возмушений возникает статистическое среднее вида $\langle T \overline{1} 1 \overline{2} 2 \overline{3} 3\rangle$, в котором операторы со своими индексами для краткости записи заменены просто этими индексами. Введением необходимого числа единичных множителей (17) осушествим разбиение исходного произведения операторов на произведение локальных групп, каждая из которых удовлетворяет вышеупомянутым требованиям и обладает отличным от нуля средним. В результате получаем

$$
\langle T \overline{1} 1 \overline{2} 2 \overline{3} 3\rangle_{0}=-\langle(T 1 \overline{2})(T 2 \overline{3})(T 3 \overline{1})\rangle_{0} \psi_{1 \overline{3}} \psi_{2 \overline{1}} \psi_{3 \overline{2}}-\langle(T 1 \overline{3})(T 3 \overline{2})(T 2 \overline{1})\rangle_{0} \psi_{1 \overline{2}} \psi_{2 \overline{3}} \psi_{3 \overline{1}} .
$$

Здесь предполагается, что операторы, заключенные в круглые скобки, обладают одинаковыми узельными индексами, например $(T 1 \overline{2})=\delta_{i_{1} j_{2}} T_{1}$, и поэтому должны усредняться совместно. Знак минус учитывает перестановки электронных операторов под знаком $T$-произведения. Так как все три группы операторов имеют отличные друг от друга узельные индексы, о чем свидетельствует нужное число $\psi$-символов, то их статистическое усреднение выполняется независимо друг от друга. Таким образом получаем

$$
\langle T \overline{1} 1 \overline{2} 2 \overline{3} 3\rangle_{0}=-\langle T 1 \overline{2}\rangle_{0}\langle T 2 \overline{3}\rangle_{0}\langle T 3 \overline{1}\rangle_{0} \psi_{1 \overline{3}} \psi_{2 \overline{1}} \psi_{3 \overline{2}}-\langle T 1 \overline{3}\rangle_{0}\langle T 3 \overline{2}\rangle_{0}\langle T 2 \overline{1}\rangle_{0} \psi_{1 \overline{2}} \psi_{2 \overline{3}} \psi_{3 \overline{1}} .
$$

Под знаком сумм и интегралов, присущих рядам теории возмущений, оба слагаемых правой части этого выражения оказываются эквивалентными и соответствующая вакуумная диаграмма третьего порядка имеет коэффициент $2 / 3 !=1 / 3$, тогда как рассмотренная выше диаграмма второго порядка - коэффищиент $1 / 2$.

Роль $\psi$-символов до сих пор состояла в разделении групп операторов, принадлежащих разным узлам, что позволяло выполнить независимым образом статистическое усреднение для каждой локальной группы операторов. После выполнения этого усреднения роль $\psi_{i j}$-символов исчерпывается и их следует устранить, заменив на $1-\delta_{i j}$. При этом $\delta_{i j}$ объединяет уже вместе статистические средние для узлов $i$ и $j$, если только это не связано с обращением в нуль интеграла перескока при совпадающих узлах. При этом возникает структура неприводимых функций Грина (или кумулянтов Кубо [7]), определенных ниже. 
В рассмотренных двух простейших примерах невозможно возникновение локальных групп операторов с числом множителей больше двух. Однако уже в четвертом порядке теории возмушений возможно возникновение групп операторов с четырьмя множителями (два оператора рождения и два уничтожения). Эти группы являются причиной появления после устранения всех нетривиальных множителей $\psi_{i j}$ одноузельных двухчастичных неприводимых функций Грина (или кумулянтов). Под тривиальными символами $\psi_{i j}$ подразумеваются те, чьи индексы совпадают с индексами интегралов перескока меж ду узлами, т.е. $\psi_{\overline{1} 1}, \psi_{\overline{2} 2} \ldots$

В высших порядках теории возмущений $n=2 m$ возникают более высокие по числу частиц одноузельные $m$-частичные неприводимые функции Грина, а также их произведения с числами частиц $m_{1}, m_{2}$ и т.д., где $m=m_{1}+m_{2}+\ldots$.

Соответствующие вклады, изображенные графически, учитывают знак и число эквивалентных диаграмм.

Таким образом, для построения диаграмм теории возмущений используются исходные электронные операторы и задача сводится к вычислению одноузельных $m$-частичных функций Грина. Только на этом втором этапе вычисления одноузельных величин используется представление электронных операторов через операторы Хаббарда, посредством которых диагонализуется оператор $H_{i \mathrm{e}}^{0}(7)$ и выполняются операции $T$-упорядочения.

Так как четвертый порядок теории возмущений содержит новый, отмеченный выше, кумулянт, рассмотрим подробнее выгисление среднего $\langle T \overline{1} 1 \overline{2} 2 \overline{3} 3 \overline{4} 4\rangle_{0}$. Введение нужного числа единичных множителей $\delta_{i j}+\psi_{i j}$ позволяет выполнить разбиение операторов на локальные группы и усреднить независимо $T$-произведения операторов, принадлежаших к разным узлам. Соберем далее под знаком сумм и интегралов эквивалентные ненулевые вклады. Имеются четыре групшы слагаемых:

$$
\begin{gathered}
3\langle T 1 \overline{2}\rangle_{0}\langle T 2 \overline{1}\rangle_{0}\langle T 3 \overline{4}\rangle_{0}\langle T 4 \overline{3}\rangle_{0} \psi_{1 \overline{3}} \psi_{1 \overline{4}} \psi_{2 \overline{3}} \psi_{2 \overline{4}} \\
-6\langle T 1 \overline{2}\rangle_{0}\langle T 2 \overline{3}\rangle_{0}\langle T 3 \overline{4}\rangle_{0}\langle T 4 \overline{1}\rangle_{0} \psi_{1 \overline{3}} \psi_{1 \overline{4}} \psi_{2 \overline{1}} \psi_{2 \overline{4}} \\
12\langle T 12 \overline{3} \overline{4}\rangle_{0}\langle T 4 \overline{1}\rangle_{0}\langle T 3 \overline{2}\rangle_{0} \psi_{1 \overline{3}} \psi_{3 \overline{2}}, \\
3\langle T 12 \overline{3} \overline{4}\rangle_{0}\langle T \overline{1} \overline{2} 34\rangle_{0} .
\end{gathered}
$$

Коэффициент перед каждым слагаемым указывает на число эквивалентных диаграмм. Все приведенные в (20) статистические средние являются одноузельными, и, следовательно, предполагается равенство всех их индексов узлов. В частности, имеem

$$
\langle T 12 \overline{3} \overline{4}\rangle_{0}=\delta_{i_{1} i_{2}} \delta_{i_{1} j_{3}} \delta_{i_{1} j_{4}}\left\langle T \tilde{c}_{\sigma_{1}}\left(\tau_{1}\right) \tilde{c}_{\sigma_{2}}\left(\tau_{2}\right) \tilde{\bar{c}}_{\sigma_{3}}\left(\tau_{3}\right) \tilde{\bar{c}}_{\sigma_{4}}\left(\tau_{4}\right)\right\rangle_{0}
$$

Здесь индекс узла операторов под знаком среднего опушен, т.к. от него это среднее не зависит.

Теперь после осушествления статистического усреднения устраним выполнившие свою роль $\psi$-символы. Учитывая их определение, для величины, стояшей под знаком соответствуюших интегралов и сумм в рассматриваемом среднем, получаем следуюшее 


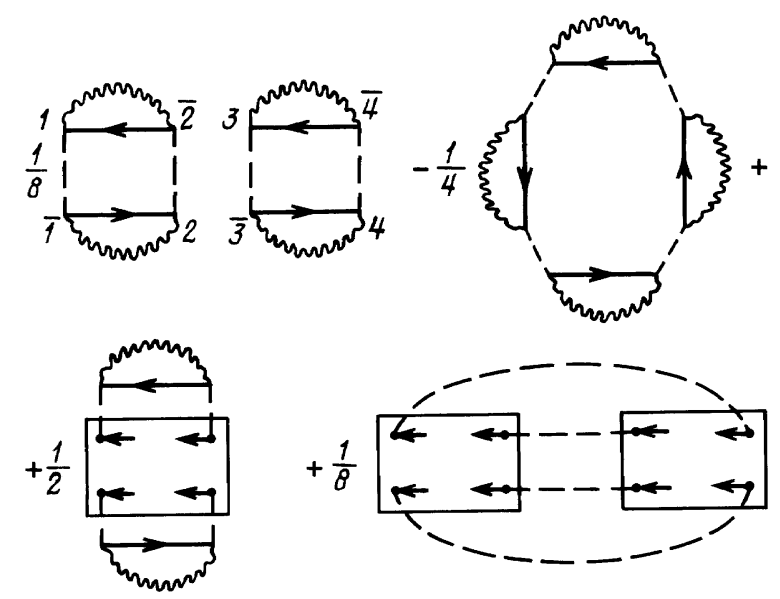

Рис. 2. Вакуумные диаграммы 4-го порядка.

окончательное выражение:

$$
\begin{aligned}
\langle T \overline{1} 1 \overline{2} 2 \overline{3} 3 \overline{4} 4\rangle_{0} \Longrightarrow & 3 \widetilde{G}^{0}(1 \mid \overline{2}) \widetilde{G}^{0}(2 \mid \overline{1}) \widetilde{G}^{0}(3 \mid \overline{4}) \widetilde{G}^{0}(4 \mid \overline{3})- \\
& -6 \widetilde{G}^{0}(1 \mid \overline{2}) \widetilde{G}^{0}(2 \mid \overline{3}) \widetilde{G}^{0}(3 \mid \overline{4}) \widetilde{G}^{0}(4 \mid \overline{1})+ \\
& +12 \widetilde{G}_{2}^{(0) \operatorname{ir}}(12 \mid \overline{3} \overline{4}) \widetilde{G}^{0}(4 \mid \overline{1}) \widetilde{G}^{0}(2 \mid \overline{3})+ \\
& +3 \widetilde{G}_{2}^{(0) \operatorname{ir}}(12 \mid \overline{3} \overline{4}) \widetilde{G}_{2}^{(0) \operatorname{ir}}(43 \mid \overline{2} \overline{1})
\end{aligned}
$$

где

$$
\begin{aligned}
& \widetilde{G}_{2}^{(0) \operatorname{ir}}(12 \mid \overline{3} \overline{4})=\delta_{i_{1} i_{2}} \delta_{i_{1} j_{3}} \delta_{i_{1} j_{4}} \widetilde{G}_{2}^{0 \mathrm{ir}}\left(\sigma_{1} \tau_{1} ; \sigma_{2} \tau_{2} \mid \sigma_{3} \tau_{3} ; \sigma_{4} \tau_{4}\right), \\
& \widetilde{G}_{2}^{(0) \operatorname{ir}}\left(\sigma_{1} \tau_{1} ; \sigma_{2} \tau_{2} \mid \sigma_{3} \tau_{3} ; \sigma_{4} \tau_{4}\right)=\left\langle T \tilde{c}_{\sigma_{1}}\left(\tau_{1}\right) \tilde{c}_{\sigma_{2}}\left(\tau_{2}\right) \tilde{\bar{c}}_{\sigma_{3}}\left(\tau_{3}\right) \tilde{\bar{c}}_{\sigma_{4}}\left(\tau_{4}\right)\right\rangle_{0}- \\
& -\left\langle T \tilde{c}_{\sigma_{1}}\left(\tau_{1}\right) \tilde{\bar{c}}_{\sigma_{4}}\left(\tau_{4}\right)\right\rangle_{0}\left\langle T \tilde{c}_{\sigma_{2}}\left(\tau_{2}\right) \tilde{\bar{c}}_{\sigma_{3}}\left(\tau_{3}\right)\right\rangle_{0}+ \\
& +\left\langle T \tilde{c}_{\sigma_{1}}\left(\tau_{1}\right) \tilde{\bar{c}}_{\sigma_{3}}\left(\tau_{3}\right)\right\rangle_{0}\left\langle T \tilde{c}_{\sigma_{2}}\left(\tau_{2}\right) \tilde{\bar{c}}_{\sigma_{4}}\left(\tau_{4}\right)\right\rangle_{0} \text {. }
\end{aligned}
$$

Отвечающие (21) диаграммы изображены на рис. 2 (с учетом общего множителя 1/4!). Эти диаграммы подобны приведенным в работах $[1,2]$, однако отличаются наличием волнистых линий, которые описывают фононные облака, присушие поляронам. Прямоугольники на рис. 2 изображают одноузельную неприводимую двухчастичную функцию Грина (или кумулянт Кубо). Она обладает сложной зависимостью от моментов времени $\tau_{i}$ :

$$
\begin{aligned}
\widetilde{G}_{2}^{0} & \operatorname{ir} \\
\quad & \left(\sigma_{1} \tau_{1} ; \sigma_{2} \tau_{2} \mid \sigma_{3} \tau_{3} ; \sigma_{4} \tau_{4}\right)=\left\langle T c_{\sigma_{1}}\left(\tau_{1}\right) c_{\sigma_{2}}\left(\tau_{2}\right) \bar{c}_{\sigma_{3}}\left(\tau_{3}\right) \bar{c}_{\sigma_{4}}\left(\tau_{4}\right)\right\rangle_{0} \times \\
& \times\left\langle\exp \left[i \bar{g}\left(p\left(\tau_{1}\right)+p\left(\tau_{2}\right)-p\left(\tau_{3}\right)-p\left(\tau_{4}\right)\right)\right]\right\rangle_{0}- \\
& -\left\langle T c_{\sigma_{1}}\left(\tau_{1}\right) \bar{c}_{\sigma_{4}}\left(\tau_{4}\right)\right\rangle_{0}\left\langle T c_{\sigma_{2}}\left(\tau_{2}\right) \bar{c}_{\sigma_{3}}\left(\tau_{3}\right)\right\rangle_{0}\left\langle T \exp \left[i \bar{g}\left(p\left(\tau_{1}\right)-p\left(\tau_{4}\right)\right)\right]\right\rangle_{0} \times \\
& \times\left\langle T \exp \left[i \bar{g}\left(p\left(\tau_{2}\right)-p\left(\tau_{3}\right)\right)\right]\right\rangle_{0}+\left\langle T c_{\sigma_{1}}\left(\tau_{1}\right) \bar{c}_{\sigma_{3}}\left(\tau_{3}\right)\right\rangle_{0}\left\langle T c_{\sigma_{2}}\left(\tau_{2}\right) \bar{c}_{\sigma_{4}}\left(\tau_{4}\right)\right\rangle_{0} \times \\
& \times\left\langle T \exp \left[i \bar{g}\left(p\left(\tau_{1}\right)-p\left(\tau_{3}\right)\right)\right]\right\rangle_{0}\left\langle T \exp \left[i \bar{g}\left(p\left(\tau_{2}\right)-p\left(\tau_{4}\right)\right)\right]\right\rangle_{0} .
\end{aligned}
$$




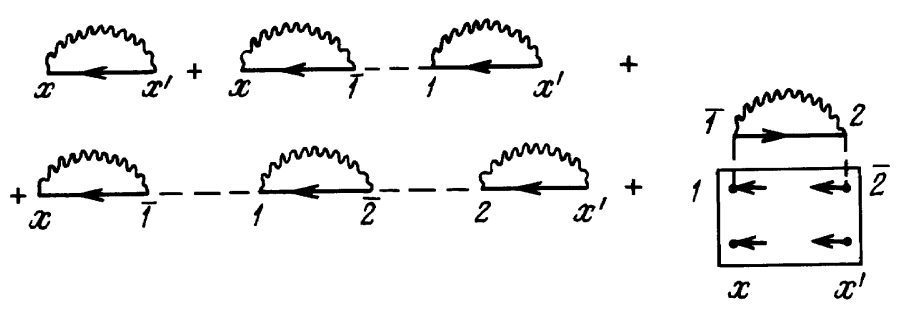

Рис. 3. Простейшие диаграммы одночастичной функции Грина.

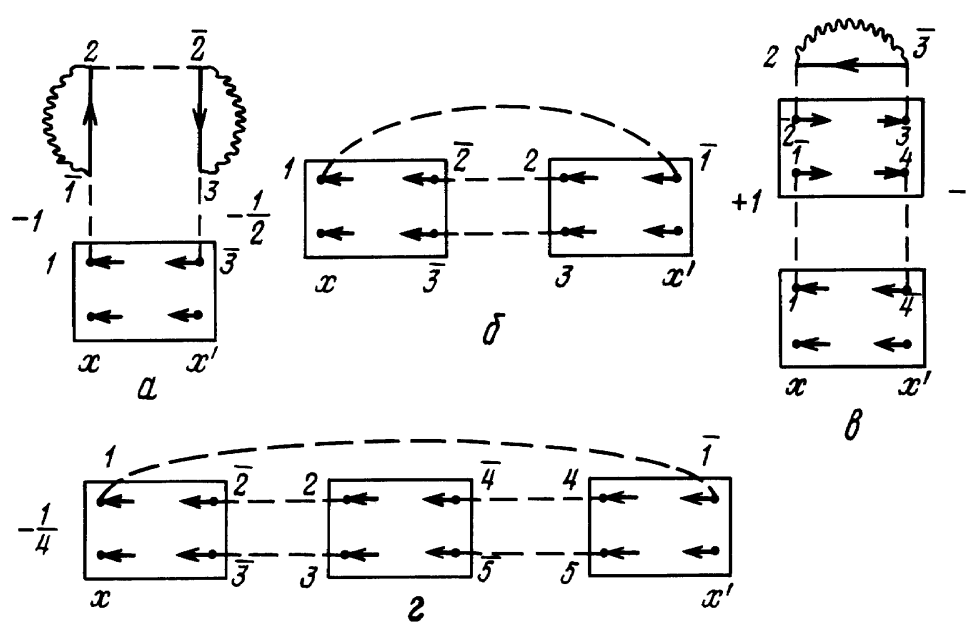

Рис. 4. Диаграммы для функции $Z(x \mid x)$.

Нетрудно убедиться в выполнении теоремы о несвязных диаграммах:

$$
\langle U(\beta)\rangle_{0}=\exp \left[\langle U(\beta)\rangle_{0}^{c}\right]
$$

где $\langle U(\beta)\rangle_{0}^{c}$ означает сумму всевозможных связных вакуумных диаграмм. Единица исключена из этого определения.

Для вычисления электронной функции Грина (9) следует обобшить предложенный метод вычисления вакуумных вкладов. На рис. 3 приведены некоторые простейшие одночастичные функции Грина.

Как было показано в работах $[1,2]$, чтобы написать уравнения Дайсона для одночастичной функции Грина, необходимо наряду с массовым оператором, равным интегралу перескока, ввести дополнительную неприводимую функцию $Z$, являющуюся суммой кумулянтов. Из определения исключаются слабосвязанные диаграммы, т.е. те, которые могут быть разделены на две части разрьвом лиш одной линии перескока. Простейшая $Z^{2}$-функция приведена на рис. 3 . Некоторые более сложные $Z$-функции изображены на рис. 4 , где диаграммы "а" и "б" отвечают 3-му, диаграмма "в" - 4-му, а диаграмма "г" 5 -му порядкам теории возмущений.

Среди диаграмм ренормированной одночастичной функции Грина имеются цепочечные графы (см. три первых на рис. 3), содержашие только нулевые одночастичные функ- 
ции Грина и интегралы перескока. Ограничение этими диаграммами приводит к приближению Хаббард I для электронного пропагатора $G^{I}$. В этом приближении пренебрегается всеми кумулянтами, т.е. функция $Z$ полагается равной нулю:

$$
G^{I}\left(x \mid x^{\prime}\right)=\widetilde{G}^{0}\left(x \mid x^{\prime}\right)+\int_{0}^{\beta} d \tau_{1} \sum_{\overline{1} 1} \widetilde{G}^{0}(x \mid \overline{1}) t(\overline{1}-1) G^{I}\left(1 \mid x^{\prime}\right) .
$$

Введем фурье-компоненты функции Грина:

$$
G\left(x \mid x^{\prime}\right)=\frac{1}{N} \sum_{\vec{k}} \frac{1}{\beta} \sum_{\omega_{n}} G_{\sigma}\left(\vec{k} \mid i \omega_{n}\right) \exp \left\{-i \vec{k}\left(\vec{x}-\vec{x}^{\prime}\right)-i \omega_{n}\left(\tau-\tau^{\prime}\right)\right\} .
$$

Тогда приближение Хаббард I имеет вид

$$
G_{\sigma}^{I}\left(\vec{k} \mid i \omega_{n}\right)=\left[\widetilde{G}_{\sigma}^{(0)-1}\left(i \omega_{n}\right)-\varepsilon(\vec{k})\right]^{-1}
$$

где величина $\varepsilon(\vec{k})$, равная энергии зонного электрона, есть фурье-образ интеграла перескока. В присутствии фононов функция Грина существенно зависит от констант $\bar{g}$ и $\omega_{0}$.

Чтобы получить уравнения Дайсона для полной одночастичной функции Грина, введем величину $\Lambda\left(x \mid x^{\prime}\right)=\widetilde{G}^{0}\left(x \mid x^{\prime}\right)+Z\left(x \mid x^{\prime}\right)$. Тогда можно написать уравнение

$$
G\left(x \mid x^{\prime}\right)=\Lambda\left(x \mid x^{\prime}\right)+\sum_{\overline{1} 1} \int_{0}^{\beta} d \tau_{1} \Lambda(x \mid \overline{1}) t(\overline{1}-1) G\left(1 \mid x^{\prime}\right) .
$$

$\mathrm{B}\left(\vec{k}, \omega_{n}\right)$-представлении при диагональности по спиновым индексам имеем

$$
G_{\sigma}\left(\vec{k} \mid i \omega_{n}\right)=\frac{\Lambda_{\sigma}\left(\vec{k} \mid i \omega_{n}\right)}{1-\varepsilon(\vec{k}) \Lambda_{\sigma}\left(\vec{k} \mid i \omega_{n}\right)} .
$$

Рассмотрим теперь функцию $Q(x)(13)$, определяющую равновесное положение фононов после сделанного унитарного преобразования. При этом среднее от самой локальной координаты фонона $q_{x}$ равно нулю. Отличные от нуля значения возникнут, если $q_{x}$ умножается на множители вида $e^{i \bar{g} p}$, входящие в операторы $\tilde{c}, \tilde{\bar{c}}$. Следовательно, следует рассмотреть возможные принадлежсности $q_{x}$ к одноузельным группам электронных и фононных операторов и определить средние вида

$$
\begin{aligned}
\left\langle T q_{x} 1 \overline{2}\right\rangle_{0}= & \left\langle T q(\tau) \tilde{c}_{\sigma_{1}}\left(\tau_{1}\right) \tilde{\tilde{c}}_{\sigma_{2}}\left(\tau_{2}\right)\right\rangle_{0}=\left\langle T c_{\sigma_{1}}\left(\tau_{1}\right) \bar{c}_{\sigma_{2}}\left(\tau_{2}\right)\right\rangle_{0} \times \\
& \times\left\langle T q(\tau) \exp \left[i \bar{g}\left(p\left(\tau_{1}\right)-p\left(\tau_{2}\right)\right)\right]\right\rangle_{0}, \\
\left\langle T q_{x} 12 \overline{3} \overline{4}\right\rangle_{0}^{\mathrm{ir}}= & \left\langle T q_{x} 12 \overline{3} \overline{4}\right\rangle_{0}-\left\langle T q_{x} 1 \overline{4}\right\rangle_{0}\langle T 2 \overline{3}\rangle_{0}-\langle T 1 \overline{4}\rangle_{0} \times \\
& \times\left\langle T q_{x} 2 \overline{3}\right\rangle_{0}+\left\langle T q_{x} 1 \overline{3}\right\rangle_{0}\langle T 2 \overline{4}\rangle_{0}+\langle T 1 \overline{3}\rangle_{0}\left\langle T q_{x} 2 \overline{4}\right\rangle_{0}, \\
\left\langle T q_{x} 12 \overline{3} \overline{4}\right\rangle_{0}= & \left\langle T c_{\sigma_{1}}\left(\tau_{1}\right) c_{\sigma_{2}}\left(\tau_{2}\right) \bar{c}_{\sigma_{3}}\left(\tau_{3}\right) \bar{c}_{\sigma_{4}}\left(\tau_{4}\right)\right\rangle_{0} \times \\
& \times\left\langle T q(\tau) \exp \left[i \bar{g}\left(p\left(\tau_{1}\right)+p\left(\tau_{2}\right)-p\left(\tau_{3}\right)-p\left(\tau_{4}\right)\right)\right]\right\rangle_{0} .
\end{aligned}
$$

Этими формулами определены новые графические элементы рис. 5 , содержащие индекс $x$. Так как все вышеприведенные величины являются одноузельными, этот индекс 

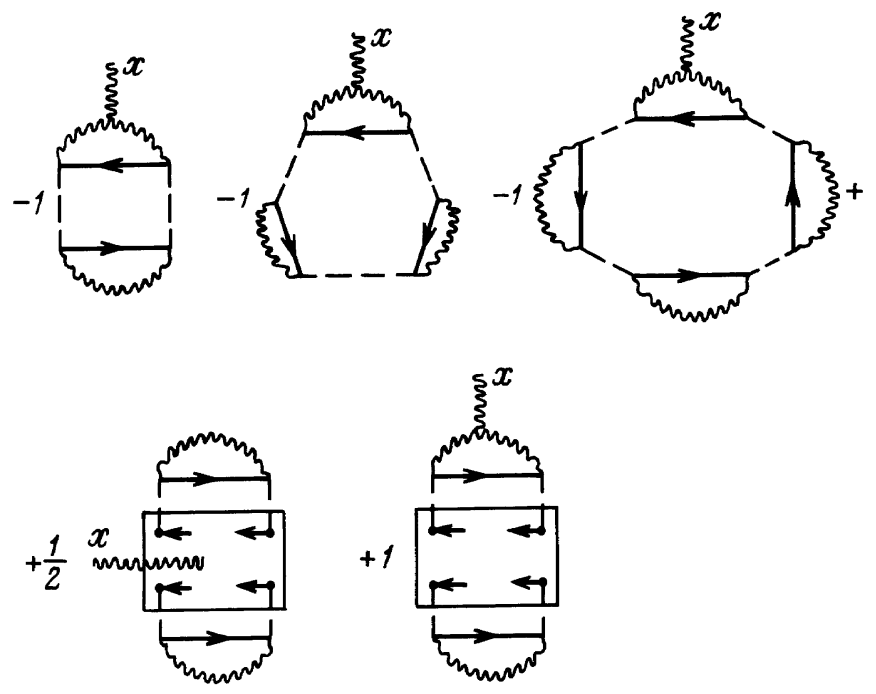

Рис. 5. Диаграммы для функции $Q(x)$.

опускается при расчете средних. На рис. 5 приведены простейшие диаграммы для функции $Q(x)$ с их коэффициентами и знаками.

В присутствии фононов неприводимая двухчастичная функция Грина усложняется из-за включения фононного оператора в различные одноузельные электрон-фононные структуры.

При вычислении функции $B_{1}\left(x \mid x^{\prime}\right)$ возникают более сложные неприводимые структуры вида

$$
\begin{aligned}
\left\langle T \widetilde{q_{x} q_{x^{\prime}}} 1 \overline{2}\right\rangle_{0}= & \delta_{x x^{\prime}} \delta_{x i_{1}} \delta_{x j_{2}}\left\langle T c_{\sigma_{1}}\left(\tau_{1}\right) \bar{c}_{\sigma_{2}}\left(\tau_{2}\right)\right\rangle_{0} \times \\
& \times\left\langle T\left(q(\tau) q\left(\tau^{\prime}\right)-\left\langle T q(\tau) q\left(\tau^{\prime}\right)\right\rangle\right) \exp \left[i \bar{g}\left(p\left(\tau_{1}\right)-p\left(\tau_{2}\right)\right)\right]\right\rangle_{0} \\
\left\langle T \widetilde{q_{x} q_{x^{\prime}}} 12 \overline{3} \overline{4}\right\rangle_{0}^{i r}= & \left\langle T\left(q(\tau) q\left(\tau^{\prime}\right)-\left\langle T q(\tau) q\left(\tau^{\prime}\right)\right\rangle_{0}\right) 12 \overline{3} \overline{4}\right\rangle_{0}-\left\langle T \widetilde{q_{x} q_{x^{\prime}}} 1 \overline{4}\right\rangle_{0}\langle T 2 \overline{3}\rangle_{0}- \\
& -\langle T 1 \overline{4}\rangle_{0}\left\langle T \widetilde{q_{x} q_{x^{\prime}}} 2 \overline{3}\right\rangle_{0}+\left\langle T \widetilde{q_{x} q_{x^{\prime}}} 1 \overline{3}\right\rangle_{0}\langle T 2 \overline{4}\rangle_{0}+\langle T 1 \overline{3}\rangle_{0}\left\langle T \widetilde{q_{x} q_{x^{\prime}}} 2 \overline{4}\right\rangle_{0}- \\
& -\left\langle T q_{x} 1 \overline{4}\right\rangle_{0}\left\langle T q_{x^{\prime}} 2 \overline{3}\right\rangle_{0}-\left\langle T q_{x^{\prime}} 1 \overline{4}\right\rangle_{0}\left\langle T q_{x} 2 \overline{3}\right\rangle_{0}+ \\
& +\left\langle T q_{x} 1 \overline{3}\right\rangle_{0}\left\langle T q_{x^{\prime}} 2 \overline{4}\right\rangle_{0}+\left\langle T q_{x^{\prime}} 1 \overline{3}\right\rangle_{0}\left\langle T q_{x} 2 \overline{4}\right\rangle_{0} .
\end{aligned}
$$

Эти новые функции имеют свое графическое изображение на рис. 6. Другая особенность функции $B_{1}$ состоит в том, что она содержит со знаком минус произведение функций $Q(x) Q\left(x^{\prime}\right)$ :

$$
B_{1}\left(x \mid x^{\prime}\right)=B\left(x \mid x^{\prime}\right)-Q(x) Q\left(x^{\prime}\right) .
$$

Некоторые простейшие графики для этой последней функции также приведены на рис. 6 . Фононная функция Грина данной системы не содержит слабосвязанных частей, соединенных одной фононной линией, вследствие чего в узлах решетки при перескоке поляронов излучается и поглошается неограниченное число фононов. Поэтому в данном подходе в обычном понимании не возникают поляризационньй оператор и соответствующее фононное уравнение Дайсона.

5 Теоретическая и математическая физика, т. 111, № 3, 1997 г. 

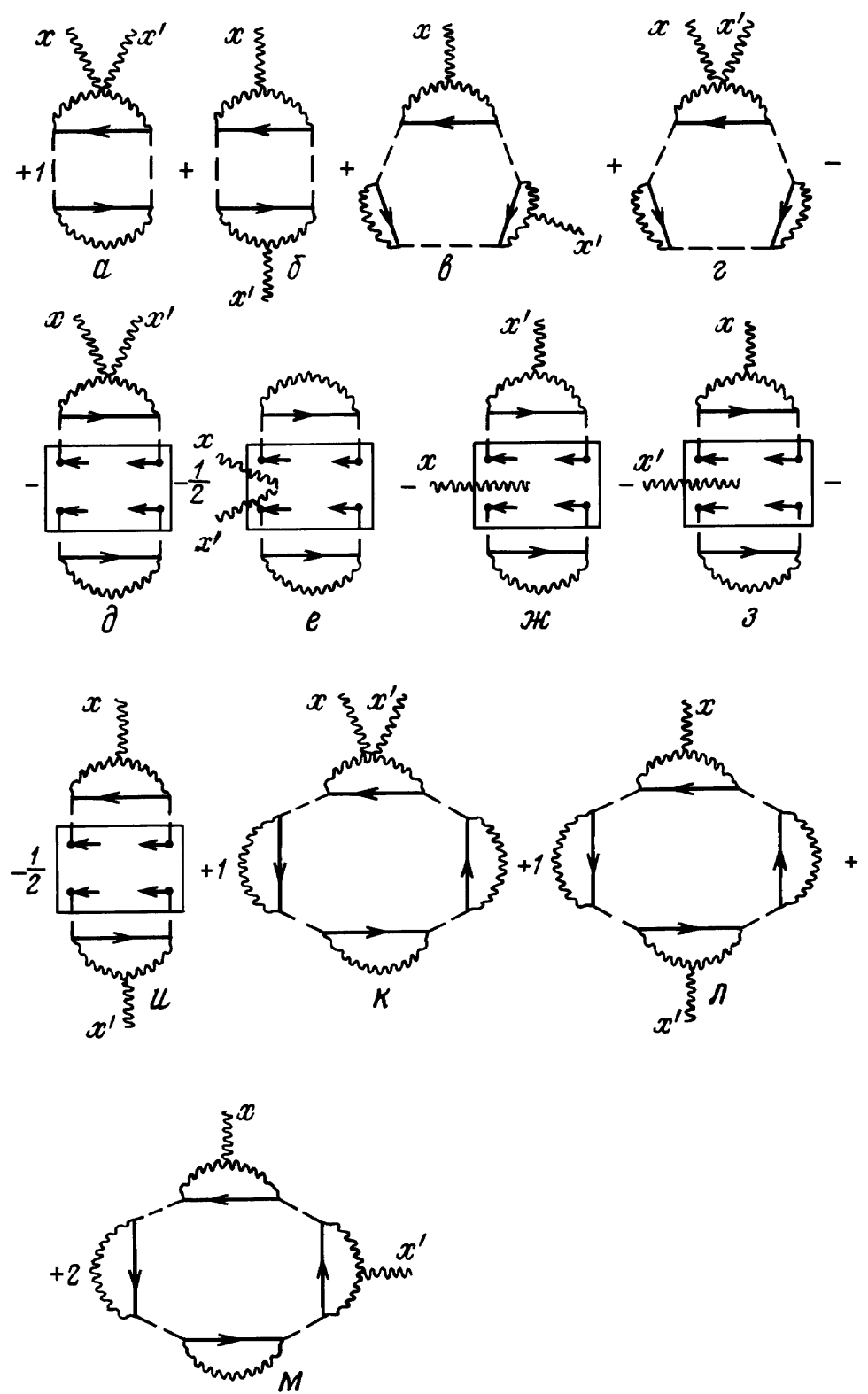

Рис. 6. Диаграммы для функции $B(x \mid x)$.

\section{ЗАКЛЮЧЕНИЕ}

Для выяснения роли фононов в установлении свойств высокотемпературных сверхпроводящих материалов необходимо дальнейшее более глубокое изучение модели Хаббарда-Холстейна, рассмотренной выше. В данной работе удается лишш установить ряд обших свойств механизма туннелирования поляронов при наличии их сильных корреля- 
ций, разработать адекватную данной задаче диаграммную технику, убедиться в справедливости теоремы о связных диаграммах и вывести уравнение Дайсона для электронной функции Грина. Установить аналогичное уравнение для фононной функции оказалось невозможным.

Работа была частично поддержана грантом фонда Сороса, предоставленного Американским физическим обшеством.

Автор пользуется случаем выразить глубокую признательность профессору Н. М. Плакиде за обсуждение некоторых аспектов работы.

\section{Список литературы}

[1] М. И. Владимир, В. А. Москаленко. ТМФ. 1990. Т. 82. С. 428.

[2] С. М. Вакару, М. И. Владимир, В. А. Москаленко. ТМФ. 1990. Т. 85. С. 248.

[3] V. A. Moskalenko, Wang Xi-Fu, Wang Zhi-Xing, Yi Xue-Xi. ТМФ. 1995. T. 103. C. 138.

[4] J. Hubbard. J. Proc. R. Soc. A. 1963. V. 276. P. 233

[5] T. Holstein. Ann. Phys. 1959. V. 8. № 3. P. 325.

[6] И. Г. Ланг, Ю. А. Фирсов. ЖЭТФ. 1962. Т. 43. С. 1843.

[7] R. Kubo. J. Phys. Soc. Japan. 1962. V. 17. № 7. P. 1100.

Поступила в редакцию 2.XII.1996 г.

\section{A. Moskalenko \\ ELECTRON-PHONON INTERACTION OF STRONG CORRELATED SYSTEMS}

The Hubbard-Holstein model after Canonical Lang-Firsov transformation is investigated. Two peculiarities of the model are taken into account: the strong electron correlations and the fact that the hopping of polarons between lattice sites are accompanied by an unrestricted number of phonons. A new diagram technique based on the classification of irreducible multi-particle Green functions is developed. A theorem about the Connected Vacuum diagrams is established and the Dyson equation for the electron Green functions is obtained. 